\title{
Echocardiographic Assessment of Cardiac Complications in Anorexia Nervosa: Correlation with the Disease Severity and the Role of Global Longitudinal Strain
}

\author{
Maria Chiara Meucci ${ }^{1}$, Gabriella Locorotondo ${ }^{1}$, Silvia Della Casa ${ }^{1}$, Filippo Crea $\mathbf{M}^{2}$ and Leonarda Galiuto ${ }^{2 *}$ \\ ${ }^{1}$ Department of Cardiovascular Sciences, Catholic University of the Sacred Heart, Italy
}

${ }^{2}$ Department of Endocrinology, Catholic University of the Sacred Heart, Italy

Submission: September 14, 2020; Published: September 21, 2020

*Corresponding author: Leonarda Galiuto, Department of Endocrinology, Catholic University of the Sacred Heart, Italy

\section{Abstarct}

a) Anorexia Nervosa (AN) is an eating disorder with devastating physical consequences. Cardiac involvement is frequent and contributes to the high morbidity and mortality rates in these patients. In our study we aimed to describe the cardiac structural and functional abnormalities occurring in AN, correlating them with the severity of the disease.

b) We enrolled 39 AN patients (mean age $22.1 \pm 7.1$ years, mean BMI $15 \pm 2.1 \mathrm{~kg} / \mathrm{m}^{2}$ ) referred to the psychiatric department of our hospital from September 2018 to June 2019. The cohort was composed by $20(51.3 \%)$ patients with extreme AN (BMI $\left.<15 \mathrm{~kg} / \mathrm{m}^{2}\right)$ and $19(48.7 \%)$ patients with non-extreme AN (BMI>15 kg/m²). All subjects underwent electrocardiogram (ECG) and two-dimensional Doppler echocardiography with speckle tracking echocardiographic (2D-STE)-derived strain imaging.

c) ECG abnormalities were similar between patients with extreme and non-extreme AN. Left ventricular (LV) mass and cardiac chamber volumes were significantly smaller in patients with extreme AN, compared to patients with non-extreme AN. Patients with extreme AN had a significantly higher prevalence of pericardial effusion (PE) and mitral valve prolapse (MVP). 2DSTE-derived strain imaging documented a subtle LV systolic dysfunction in 21(53.8\%) patients, despite a normal LV ejection fraction (LVEF) and without significant differences between the two sub-groups. Parameters of diastolic function were within the normal range.

d) LV remodeling, PE and MVP correlated with the severity of the disease, on the contrary ECG abnormalities and parameters of LV systolic function did not. 2DSTE may uncover an early LV dysfunction in these patients, undetected by conventional echocardiography.

Keywords: Anorexia nervosa; Eating disorders; Cardiovascular diseases; Echocardiography; Ejection fraction; Left ventricular mass; Sudden cardiac death; Global longitudinal strain

Abbreviations: AN: Anorexia Nervosa; BMI: Body Mass Index; ED: Eating Disorders; ECG: Electrocardiogram; GLS: Global Longitudinal Strain; HR: Heart Rate; IVRT: Isovolumic Relaxation Time; LAV: Left Atrial Volume; LLN: Lower Limit Of Normal; LVEF: Left Ventricular Ejection Fraction; LVEDV: Left Ventricular End-Diastolic Volume; LVESV: Left Ventricular End-Systolic Volume; LVM: Left Ventricular Mass; MVP: Mitral Valve Prolapse; PE: Pericardial Effusion; SCD: Sudden Cardiac Death; 2D-STE: Two-Dimensional Speckle-Tracking Echocardiography.

\section{Introduction}

Anorexia Nervosa (AN) is a psychiatric disorder with devastating physical consequences. It is the most frequent eating disorders (ED), mainly affecting young women (10:1 female-male ratio). The incidence of $\mathrm{AN}$ has been reported to be 270 to 100.000 person-years in adolescents, although it is often underestimated [1]. Its prevalence varies between $0.9 \%$ and $3 \%$ in women and between $0.16 \%$ and $0.3 \%$ in men [2]. Of note, AN has the high est mortality rate of any psychiatric disorder, with 5.1 deaths per 1000 person years [3].

According to the DSM-5 criteria [4], the diagnosis of AN requires the presence of all the following criteria:

a) Persistent restriction of energy intake leading to significantly low body weight; 
b) Either an intense fear of gaining weight or a persistent behaviour that interferes with weight gain through restrictive eating only (anorexia nervosa restricting type, AN-R) or restrictive behaviour accompanied by episodes of binge eating followed by purging (anorexia nervosa binge purging type, AN-BP);

c) Disturbance in the way one's body weight or shape is experienced, undue influence of body shape and weight on selfevaluation, or persistent lack of insight regarding the seriousness of the condition.

d) The reduction of energy intake induces malnutrition and a series of metabolic changes leading to potentially lifethreatening somatic complications, including cardiac abnormalities, endocrine and bone impairment, electrolyte disturbances and immunodepression [5].

Among medical complications, cardiac structural, functional and rhythm-type changes are frequent, reaching $80 \%$ of cases in some reports [6,7]. Although frequently asymptomatic and undetected, the prognostic relevance of cardiac changes in AN has been clearly demonstrated [8,9]. Indeed, cardiovascular abnormalities are associated with an increased risk of sudden cardiac death (SCD) mostly triggered by ventricular arrhythmias [10], causing up to $30 \%$ of the mortality in AN $[11,12]$.

Therefore, expanding our knowledge on the cardiac consequences of $\mathrm{AN}$ is of crucial importance, in order to shed more light on these hidden but potentially deadly complications of the disease. Particularly, accurate identification of high-risk patients and early diagnosis of the cardiac involvement may have relevant therapeutic and prognostic implications. Twodimensional speckle tracking echocardiographic (2DSTE)derived strain imaging is an emerging technique that allows the quantitative characterization of LV systolic function and has been shown to have a greater sensitivity for the detection of LV dysfunction rather than conventional echocardiography in several clinical settings [13].

In this study, we aimed to describe the cardiac structural, functional and rhythm-type changes occurring in AN patients correlating them with the severity of the disease. Moreover we firstly applied the 2DSTE-derived strain imaging for the assessment of LV function in patients with AN.

\section{Methods}

\section{Participants}

We prospectively enrolled 39 patients referred to the psychiatric department of the Fondazione Policlinico Universitario Agostino Gemelli IRCCS (Rome, Italy) with a diagnosis of AN from September 2018 to June 2019. All patients fulfilled the DSM-V criteria for AN [4]. Body mass index (BMI) was obtained as a parameter of the severity of AN according to the classification reported by the DSM-V [3]. The level of severity was defined as mild-tomoderate for BMI ranging from $17 \mathrm{~kg} / \mathrm{m}^{2}$ to $16 \mathrm{~kg} / \mathrm{m}^{2}$, severe for BMI ranging from 15.99 to $15 \mathrm{~kg} / \mathrm{m}^{2}$ and extreme for BMI under $15 \mathrm{~kg} / \mathrm{m}^{2}$. The mean value of BMI in our cohort was $15 \pm 2.1 \mathrm{~kg} / \mathrm{m}^{2}$, therefore patients were finally classified in two subgroups: patients with extreme $\mathrm{AN}\left(\mathrm{BMI}<15 \mathrm{~kg} / \mathrm{m}^{2}\right)$ and patients with non-extreme AN (BMI>15 kg/m²)

All patients underwent cardiological examination, 12 leads electrocardiogram (ECG) and two-dimensional Doppler echocardiography at the time of the enrollment. Written informed consent was obtained from all the study participants or their guardians. All procedures performed were in accordance with the ethical standards of our institutional committee and with the 1964 Helsinki declaration and its later amendments or comparable ethical standards.

\section{Electrocardiogram}

Standard 12-lead Electrocardiograms (ECG) were performed in the supine position at a paper speed of $25 \mathrm{~mm} / \mathrm{s}$ and an amplitude of $10 \mathrm{mV}$. PR, QRS and QT intervals were measured manually with calipers. Bazett's formula was used to calculate the corrected QT interval: QTc = QT (in ms) divided by the square root of the preceding R-R interval in seconds. QT prolongation was defined as QTc interval longer than $470 \mathrm{~ms}$ [14].

\section{Echocardiographic examination}

A transthoracic M-mode, 2D, Doppler and speckle tracking echocardiographic examination was performed using a Toshiba Artida ultrasound machine. The study was performed with the subject lying on his left side with the head of the bed elevated by near $30^{\circ} \mathrm{C}$. Using M-mode imaging in the parasternal short-axis view, left ventricular mass (LVM) was calculated by the Devereux formula [15]: $\mathrm{LVM}=0.80 \times 1.04 \times(\mathrm{IVS} d+\mathrm{LVIDd}+\mathrm{PWTd})^{3}-\mathrm{LVIDd}^{3}+0.6$ (g), where IVSd is the interventricular septal thickness, LVDd is the largest LV internal dimension at end-diastole, and PWTd is the posterior wall thickness.

In the apical views left ventricular end-diastolic volume (LVEDV), left ventricular end-systolic volume (LVESV) and left atrial volume (LAV) were calculated according to American Society of Echocardiography guidelines [15]. Then LV global systolic function was assessed by measuring the left ventricular ejection fraction (LVEF), using the following formula [16]: $\operatorname{LVEF}(\%)=$ LVEDV- LVESV / LVEDV. The mean values of LVM, LVEDV and LVESV in our cohort were compared to the reference lower limits of normal (LLN) in women reported by the American Society of Echocardiography (67g for LVM, 61 ml for LVEDV, 21ml for LVESV) [16].

Global longitudinal strain (GLS) was calculated using the two-dimensional speckle-tracking echocardiography (2D-STE) in order to further characterize LV systolic function. Strain describes the fractional changes in the dimension of a myocardial fiber. In 
particulary, GLS describes the relative length change between end-diastole and end-systolic myocardial fiber (16): GLS (\%) = MLs-MLd/MLd; where ML is myocardial length at end-systole (MLs) and end-diastole (MLd).

Because MLs is smaller than MLd, GLS is a negative number. Increases in the absolute value mean a better LV systolic function.
Echocardiographic images of the apical four- and two-chamber views were acquired. GLS values at the apical, midventricular, and basal levels were generated and GLS was calculated as an average of the segments from the two views using a validated protocol (Figure 1) [16]. The values were then compared to the reference LLN of $-18 \%$, defined according to the software package used [16].
A

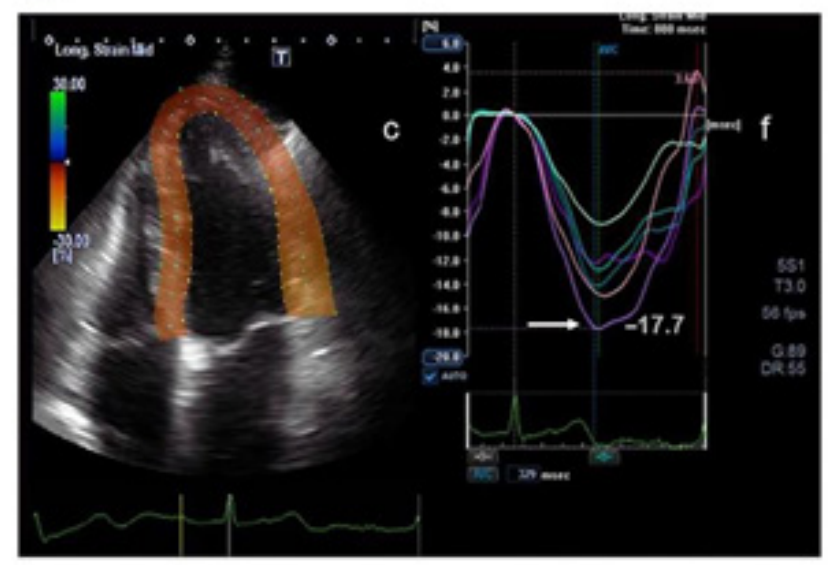

B

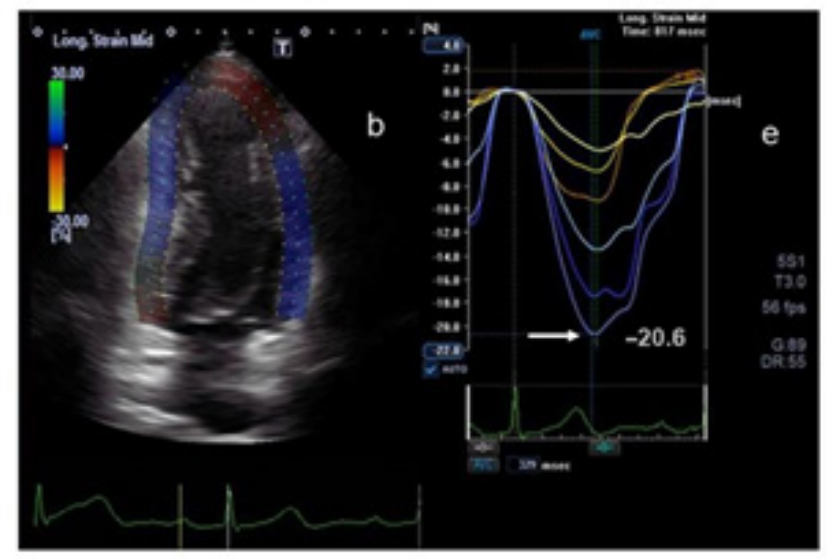

Figure 1: Apical four chamber (a) and two-chamber (b) views and the corresponding longitudinal strain curves.

Regional strain is graphically presented by 6 different color-coded curves. The peak of the average curve of all the segments (indicated by the white arrow) is considered the global longitudinal strain.

Pulsed Doppler transmitral flow velocity profile and tissue Doppler imaging were performed to evaluate the LV diastolic function. Pulsed Doppler transmitral flow velocity profile was obtained from the apical four-chamber view and the sample volume was positioned just below the mitral valve leaflets. The following parameters were evaluated: peak transmitral flow velocity in early diastole (peak E), peak transmitral flow velocity in late diastole (peak A) and E/A ratio [17]. Then Tissue Doppler imaging of diastolic velocities of the basal lateral segment and the basal interventricular septum in the apical four-chamber views were measured. The sample volumes were placed at the center of the myocardial segments. The pulsed tissue Doppler imaging of a chosen segment is characterized by a myocardial systolic wave $\left(S^{\prime}\right)$ and two diastolic waves, early (E') and atrial $\left(A^{\prime}\right)$. We measured the ratio of transmitral $E$ and the $E^{\prime}$ wave (E/E' ratio) [17].

Finally the isovolumic relaxation time (IVRT), that is the time interval between the end of the aortic ejection and the beginning of ventricular filling, was obtained from the simultaneous recording in spectral Doppler mode of the aortic and mitral flows [17]. All the parameters were measured during three consecutive cardiac cycles and the average value was calculated.

\section{Statistical Analysis}

Continuous variables were compared using an unpaired Student's t-test as appropriated and data were expressed as mean \pm standard deviation (SD). Categorical data were expressed as percentages and were evaluated using the Pearson $c^{2}$ test or Fisher exact test, as appropriate. A p value $<0.05$ was considered statistically significant. All analyses were performed using SPSS version 20 (SPSS Inc., Chicago, IL, USA).

\section{Results}

We enrolled 39 patients with AN, 38 women and 1 men. Their age ranged from 13 to 42 years, with a mean value of $22.1 \pm 7.1$ years. The average values of weight and BMI were $39.8 \pm 7.2 \mathrm{~kg}$ and $15 \pm 2.1 \mathrm{~kg} / \mathrm{m} 2$, respectively. $20(51.3 \%)$ patients were affected by non-extreme AN and 19 (48.7\%) patients by extreme AN. The subgroup of nonextreme AN was composed by 14 (35.9\%) 
patients with mild-to moderate AN and $6(15.4 \%)$ patients with severe AN. In 17 (43.5\%) subjects the disease onset was within the 2 years before the enrollment, in the other 22 (56.5\%) patients it was earlier than 2 years. Table 1 shows the main clinical characteristics of patients with extreme AN and non-extreme AN.

Table 1: Clinical data of the overall population and according to disease severity (extreme AN and nonextreme AN).

\begin{tabular}{|c|c|c|c|c|}
\hline Clinical Characteristics & Total $(\mathrm{N}=39)$ & Extreme AN $(\mathrm{N}=19)$ & Non-Extreme AN $(\mathrm{N}=20)$ & $\mathbf{P}$ \\
\hline Age (years) & $22.1 \pm 7.1$ & $24 \pm 8.4$ & $20.2 \pm 5.4$ & n.s. \\
\hline Female sex, $\mathrm{n}(\%)$ & $38(97)$ & $18(94.7)$ & $20(100)$ & n.s. \\
\hline Weight (kg) & $39.8 \pm 7.2$ & $34.1 \pm 4$ & $45.2 \pm 5$ & $<0.001$ \\
\hline BMI $\left(\mathrm{kg} / \mathrm{m}^{2}\right)$ & $15 \pm 2.1$ & $13.1 \pm 1$ & $16.8 \pm 1$ & $<0.001$ \\
\hline Duration of disease $<2$ years, $n,(\%)$ & $17(43.5)$ & $11(57.9)$ & $6(30)$ & n.s. \\
\hline
\end{tabular}

Legend: BMI: body mass index; ns: not significant ( $p$-value $>0.05$ ).

\section{ECG abnormalities}

The most common ECG abnormality documented was sinus bradycardia, affecting $22(56.4 \%)$ patients. The mean value of the heart rate (HR) was $59 \pm 10.5$ beats/minutes. A firstdegree atrioventricular block (AV block) and an incomplete intraventricular conduction delay were found in $1(2.6 \%)$ and $7(17.9 \%)$ patients respectively, with no cases of more severe conduction block. Furthermore 1 (2.6\%) patient presented a QT prolongation. The average length of the QTc interval was $397.7 \pm 24.5$ milliseconds. ECG changes did not exhibit significant differences between patients with extreme AN and non-extreme AN (Table2).

\section{Echocardiographic findings in the acute phase}

The average values of LVEDV and LVESV were $63.05 \pm 13.3 \mathrm{ml}$ and $23.1 \pm 5.5 \mathrm{ml}$, respectively (Table 3 ). In the subgroup of extreme AN LV volumes were below the reference LLN and significantly lower as compared to the subgroup of non-extreme AN (LVEDV: $56.4 \pm 13.3 \mathrm{ml}$ vs $69.4 \pm 10.1 \mathrm{ml}, \mathrm{p}=0,001$; LVESV: $20.7 \pm 5.4 \mathrm{ml}$ vs $25.4 \pm 4.6 \mathrm{ml}, \mathrm{p}=0,006$ ) (Figure $2 \mathrm{~A}-\mathrm{B}$ ). Similarly, the LAV of patients with extreme AN was significantly smaller as compared to patients with non-extreme AN $(27.1 \pm 5.6 \mathrm{ml}$ vs $33.4 \pm 6.5 \mathrm{ml}$, $\mathrm{p}=0,03$ ) (Figure $2 \mathrm{C}$ ). Both in the extreme and nonextreme AN subgroups, cardiac chamber volumes did not exhibit significant differences between patients with the disease onset before or within two years from the time of the enrollment (Figure $3 \mathrm{~A}-\mathrm{B}$ ). Patients with extreme AN had a mean value of $\mathrm{LV}$ mass that is below the LLN and numerically lower in comparison to patients with nonextreme AN, with a trend toward statistical significance $(65.9 \pm 14.6$ vs $76.5 \pm 18.7 \mathrm{ml}, \mathrm{p}=0,057)$.
A

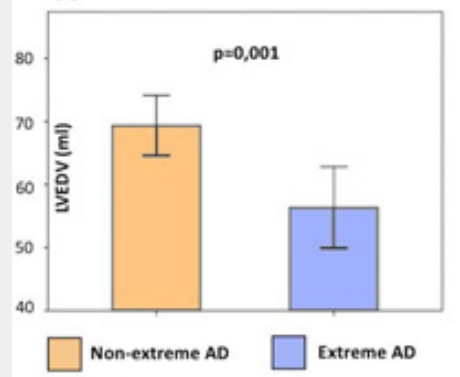

B

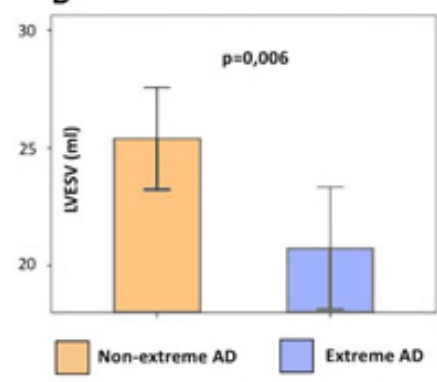

C

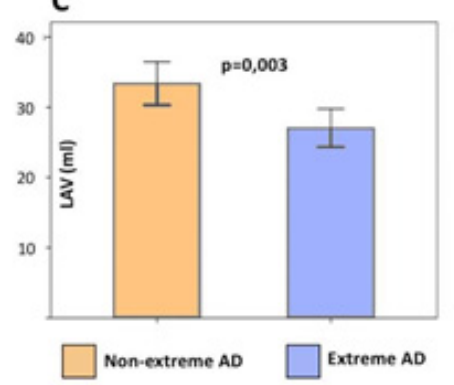

Figure 2: Comparison of cardiac chamber volumes in patients with extreme and non-extreme AN.

All patients had a normal LV ejection fraction (EF) with an average value of $63.6 \pm 3.3 \%$. However, using 2D-STEderived strain imaging, a mild LV systolic impairment has been documented in
$21(53.8 \%)$ patients of the overall population. Particularly, an abnormal GLS was found in 12 (63\%) patients with extreme AN and $9(46 \%)$ with nonextreme AN (Figure 4). GLS mean value 
in the overall population was of $-17 \pm 2.5 \%$, with no significant differences between patients with extreme AN and non-extreme AN $(-16.8 \pm 2.6 \%$ vs $-17.3 \pm 2.4 \%$, p=ns). Parameters of diastolic

function (E/A, E/E' and IVRT) were within the normal range and comparable among the two sub-groups.

Table 2: Electrocardiographic findings of the overall population and according to disease severity (extreme AN and non-extreme AN).

\begin{tabular}{|c|c|c|c|}
\hline ECG Findings & Total (N=39) & Extreme AN (N=19) & Non-Extreme AN (N=20) \\
\hline Sinus bradycardia, $\mathrm{n}(\%)$ & 22 & $10(52.6)$ & $12(60)$ \\
\hline First degree AV block, $\mathrm{n}(\%)$ & $1(2.6)$ & $0(0)$ & $1(5)$ \\
\hline $\begin{array}{c}\text { Incomplete intraventricular conduction delay, } \mathrm{n} \\
(\%)\end{array}$ & $7(17.9)$ & $5(26.3)$ & $2(10)$ \\
$\mathrm{ns}$ \\
\hline QTc prolongation, $\mathrm{n}(\%)$
\end{tabular}

Legend: AV block: atrioventricular block; ns: not significant ( $p$-value $>0.05$ )

Table 3: Echocardiographic findings of the overall population and according to disease severity (extreme AN and non-extreme AN) in the acute phase.

\begin{tabular}{|c|c|c|c|c|}
\hline Echocardiographic Findings & Total $(\mathrm{N}=39)$ & Extreme AN $(\mathrm{N}=19)$ & Non-Extreme AN ( $\mathrm{N}=20)$ & $\mathbf{P}$ \\
\hline LVEDV, ml & $63.05 \pm 13.3$ & $56.4 \pm 13.3$ & $69.4 \pm 10.1$ & 0.001 \\
\hline LVESV, ml & $23.1 \pm 5.5$ & $20.7 \pm 5.4$ & $25.4 \pm 4.6$ & 0.006 \\
\hline LVEF, \% & $63.6 \pm 3.3$ & $63.5 \pm 3.8$ & $63.7 \pm 2.8$ & ns \\
\hline GLS, \% & $-17 \pm 2.5$ & $-16.8 \pm 2.6$ & $-17.3 \pm 2.4$ & ns \\
\hline LAV, ml & $30.3 \pm 6.8$ & $27.1 \pm 5.6$ & $33.4 \pm 6.5$ & 0.003 \\
\hline LVM, gr & $71.4 \pm 17.5$ & $65.9 \pm 14.6$ & $76.5 \pm 18.7$ & ns \\
\hline $\mathrm{E} / \mathrm{A}$ & $2 \pm 0.6$ & $2 \pm 0.7$ & $2.2 \pm 0.5$ & ns \\
\hline $\mathrm{E} / \mathrm{E}^{\prime}$ & $6.1 \pm 1.1$ & $6.4 \pm 1.2$ & $5.8 \pm 0.9$ & ns \\
\hline IVRT, ms & $55 \pm 8.3$ & $54 \pm 9.7$ & $56 \pm 6.9$ & ns \\
\hline MVP, n (\%) & $6(15.4)$ & $6(31.6)$ & $0(0)$ & 0.006 \\
\hline PE, n (\%) & $16(41)$ & $11(57.9)$ & $5(25 \%)$ & 0.037 \\
\hline
\end{tabular}

Legend: IVRT: isovolumic relaxation time; LAV: left atrial volume; LVEDV: left ventricular end-diastolic volume; LVESV: left ventricular end-systolic volume; LVEF: left ventricular ejection fraction; LVM: left ventricular mass; MVP: mitral valve prolapse; PE: pericardial effusion.
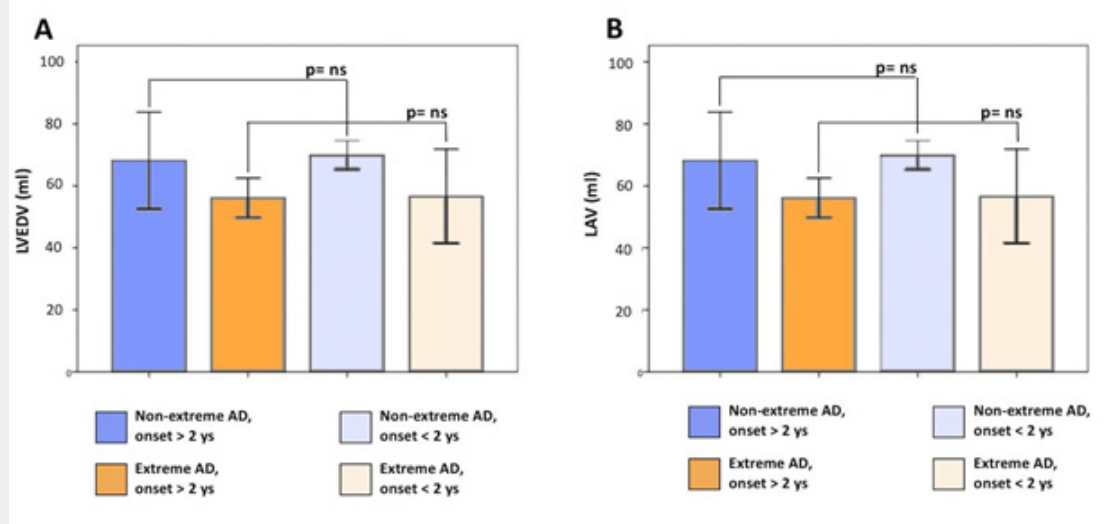

Figure 3: Comparison of cardiac chamber volumes in patients with disease onset before or within 2 years from the enrollment. 


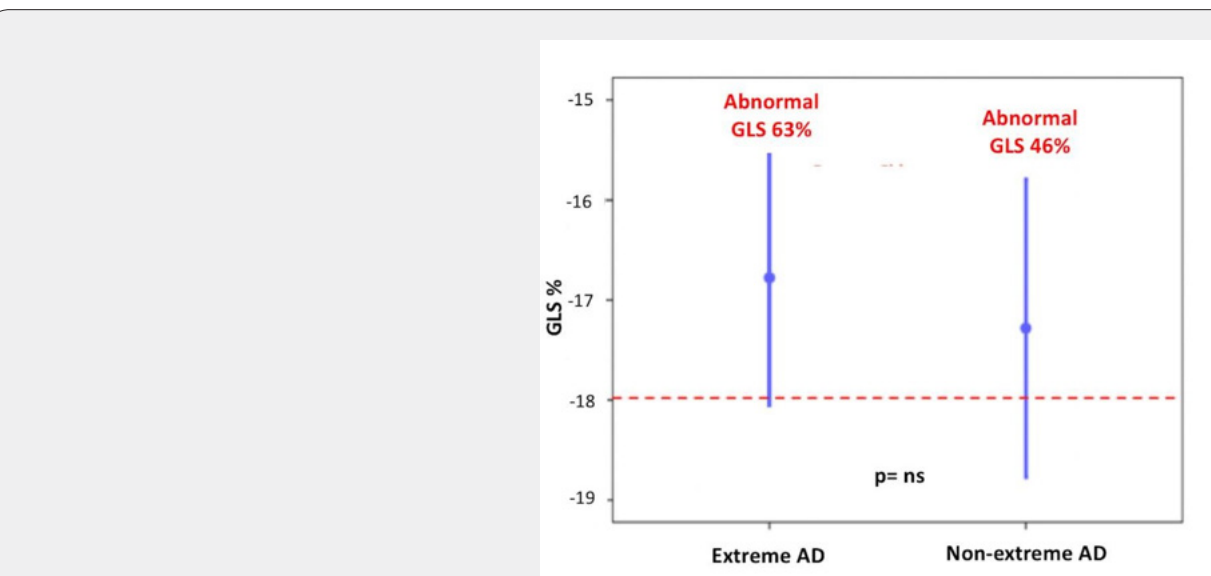

Figure 4: Prevalence of abnormal GLS in patients with extreme and non-extreme AN.

In $6(31.6 \%)$ patients with extreme AN a mitral valve prolapse (MVP) was detected, while there were no cases of MVP in the subgroup of non-extreme AN $(p=0,006)$. In all patients with MVP only a trivial to mild valvular regurgitation was found Finally 16 (41\%) patients in the overall cohort had a trivial to small amount of pericardial effusion (PE), more frequently in patients with extreme AN, as compared to non-extreme AN (57.9\% vs $25 \%$, $\mathrm{p}=0,006)$.

\section{Discussion}

The present study investigated the cardiac structural and functional changes occurring in AN. The major findings of our investigation were:

a) LV remodeling, PE and MVP correlated with the severity of the disease, on the contrary ECG abnormalities and echocardiographic parameters of LV systolic function did not.

b) 2DSTE-derived strain imaging documented a subtle LV systolic dysfunction in about half of the patients with AN, despite a normal LVEF.

AN is a chronic and severe eating disorder, most commonly affecting female adolescents or young women. It has reported to be the third most prevalent chronic disease in adolescent girls [18].

The critically limited food intake and neuroendocrine aberrations occurring in AN have crucial consequences for the general health of these patients, leading to complications in almost every organ of the body [5]. Of note, patients with AN have a mortality rate for all causes of death six times higher than the general population [19] and the highest mortality rate of any psychiatric disorder [3]. Among medical complications caused by AN, cardiac complications are often the focus of many studies, in the light of their prognostic relevance. Indeed $80 \%$ of AN patients are affected by a cardiac complication $[6,7]$ and SCD secondary to arrhythmias is often the cause of non-suicide- related deaths in these patients [10-12].

However, AN patients are not only compromised physically, but psychologically and socially as well. This complex interaction makes the disease very difficult to manage effectively and implies the need of a multidisciplinary approach. The disease is often denied by the patients and unrecognized by their families, leading to late diagnosis and treatment. Of interest, a recent study investigating the facilitators of the initial contact with professional health providers in patients with ED found that physical health concerns are a primary facilitator for seeking clinical care [20]. Although AN patients are often "not interested" to the health of their body, in our experience they take into serious account their heart shape and function. Thus, bringing these patients to focus on their heart health might produce a more effective engagement in their multidisciplinary care.

The most common ECG abnormalities reported in AN is sinus bradycardia. In our cohort it was found in $56.4 \%$ of the patients, similarly to previous studies [5]. Bradycardia may be in relation with an increased vagal tone and a concomitant decrease in sympathetic activation [3]. Interestingly, in the present study the prevalence of bradycardia and the mean values of HR were similar between patients with extreme AN and non-extreme AN. A possible explanation is that bradycardia usually represents a physiological adaptation to starvation and weight loss, rather than a disease of the cardiac conduction system. Indeed, bradycardia is reversible following nutritional treatment and significant conduction blocks in AN patients are extremely rare [5].

An additional relevant abnormality described on the ECG of anorectic patients is the prolongation of QT interval, that is due to defects in ventricular repolarization. In the presence of a prolonged QT interval, early post depolarization may determine ectopic ventricular beats that trigger ventricular arrhythmias, potentially culminating in SCD. Of note, in a recent large study 
on patients with ED, the QTc was normal in most patients with AN but was longer in AN-BP than in AN-R and was the longest in patients with bulimia nervosa, emphasizing the role of purging in QT prolongation, probably due to electrolytes abnormalities [21]. Therefore, when a prolonged QT is detected in a patients with AN, it should not summarily be ascribed to AN, but rather an extensive search for secondary causes should be pursued, including electrolytes abnormalities and psychotropic medications [5]. In our cohort we found only one case of prolonged QT (defined as QTc interval higher than $470 \mathrm{~ms}$ ), occurred in a patient with extreme AN and without other potential causes identified.

A reduced cardiac mass and smaller chamber volumes have been demonstrated in patients with AN compared to controls by previous echocardiographic studies [6,8,22,23] A postulated mechanism for the cardiac structural remodeling observed in AN is vagal hyperactivity, seen as an attempt to conserve energy, leading to changes in both contractility and preload, which then result in cardiac atrophy [8]. On the other hand, De Simone et al. [6] found that the loss of left ventricular mass in women with AN was not the same as that seen in underweight women without starvation. Thus, the specific mechanisms of remodeling seen in AN are not yet fully understood. In this study we showed a significant reduction of cardiac chamber volumes (LVEDV, LVESV, LAV) in patients with extreme AN, in comparison to patients with non-extreme AN. Similarly, the LVM is lower in patients with extreme AN, as compared to non-extreme AN, without reaching the statistical significance. On the other hand, in our anorectic sample cardiac chamber sizes did not correlate with the duration of the disease, evaluated as onset before or within two years from the enrollment.

As expected and showed in other studies $[8,9]$, all subjects disclose a normal LV ejection fraction. However, using 2DSTEderived strain imaging, we reported the novel finding of a high prevalence rate of abnormal GLS (53.8\%), showing a subtle LV contractile impairment in these patients. Of importance, this is the first study to characterize LV function with 2DSTE-derived strain imaging in anorectic subjects, apart from one small-size study by Morris et al. In contrast with our data, Morris described a normal global LV function in AN patients and a reduced regional function of LV apex in the subgroup of patients with AN-BP [24]. On the other hand, Galetta et al, using tissue Doppler imaging (TDI), reported a decrease of the LV peak systolic velocities in AN subjects compared to controls [25], supporting the hypothesis of an early stage of LV systolic dysfunction in these patients, undetected by conventional echocardiography. Nevertheless further studies need to establish the real clinical and prognostic value of 2DSTEderived strain imaging in these patients.

The abnormalities of LV function in AN patients are likely related to the changes of the myocardial wall structural properties. Indeed postmortem examination of hearts from animals and humans dying of starvation has demonstrated marked histology histologic abnormalities, including fragmentation of myofibrils, replacement fibrosis, widened interstitial spaces, due to edema fluid and cellular infiltration by mononuclear cells and marked loss of muscle fibers $[26,27]$. This kind of abnormalities could negatively affect left ventricular function by reducing the compliance as a result of "starvation edema" and by reducing the myocardial contractility as a result of myocardial fiber atrophy.

Doppler parameters of diastolic function were within the normal range in our cohort, in accordance to previous data reported by Galetta et al. [25]. This finding could seem paradoxical because diastolic dysfunction usually precedes systolic dysfunction. However it can be explained by the low diagnostic value of Doppler imaging in evaluating diastolic function in this condition, that which can be influenced by multiple interrelated factors including HR, blood pressure, LV dimension and mass.

Pericardial effusion (PE) is a frequent echocardiographic finding in patients with AN, with prevalence rate varying widely from 20 to $70 \%$ [28]. In our study $41 \%$ of patients had silent $\mathrm{PE}$, reaching the prevalence of $57.9 \%$ in patients with extreme AN. Similarly, Kastner et al reported a 34\% prevalence of PE in a cohort of 173 anorectic patients [28]. The presence of PE was associated with lower BMI, together with more prominent low-T3 syndrome and longer duration of hospitalization.

MVP is a common valve pathology, with a prevalence of $0.6-2.4 \%$ in the general population and between 33 and $62 \%$ in patients with AN [6]. In our study a MVP is found in $15.4 \%$ patients and is significantly associated with extreme AN, with a prevalence of $31.6 \%$ in this sub-group. MVP is rarely associated to significant mitral regurgitation in AN. However, the importance of MVP detection results from its association with arrhythmias and SCD (30), even if this association has not been fully documented in AN.

Cardiovascular complications of AN, particularly the decrease of LV mass and chamber volumes along with electrical abnormalities are potentially fatal, but reversible in most cases $[8,9]$. Early diagnosis of cardiac damage, prompt treatment and refeeding and correct cardiac monitoring are essential in the management of these patients. Therefore ECG and twodimensional Doppler echocardiography should be routinely performed in AN. In addition, 2D-STE is a promising modality that may be useful for identifying those AN patients who are at increased risk of developing significant cardiac dysfunction.

If specific nutritional supplement might be indicated to prevent cardiac damage in subjects that refuse the refeeding remains to be demonstrated and it is a domain of intriguing novel investigations. The major limitations of the present study are the small sample size and the lack of a control group. However this is related to the aim of our study that was to investigate the correlation between cardiac changes and the severity of the disease, rather than demonstrate their existence in comparison to healthy subjects. 


\section{Conclusion}

In this cohort study we demonstrate that LV remodeling, PE and MVP correlated with the severity of the disease, on the contrary ECG abnormalities and echocardiographic parameters of LV systolic function did not. Moreover, using 2DSTE-derived strain imaging we documented a subtle LV systolic dysfunction in about half of the patients, despite a normal LVEF. Future researches are needed to establish the real clinical and prognostic value of 2DSTE-derived strain imaging in these patients.

\section{Acknowledgement}

The authors wish to acknowledge Federica Palma, cardiac sonographer, for her contribution in the cardiac imaging of these patients.

\section{References}

1. Keski-Rahkonen A, Hoek HW, Susser ES, Linna MS, Sihvola E, et al. (2007) Epidemiology and course of anorexia nervosa in the community. Am J Psychiatry 164(8): 1259-1265.

2. Miller KK, Grinspoon SK, Ciampa J, Hier J, Herzog D, et al. (2005) Medical findings in outpatients with anorexia nervosa. Arch Intern Med 165(5): 561-566.

3. Arcelus J, Mitchell AJ, Wales J, Nielson S (2011) Mortality rates in patients with anorexia nervosa and other eating disorders. A metaanalysis of 36 studies. Arch Gen Psychiatry 68(7): 724-731.

4. American Psychiatric Association (2013) Diagnostic and statistical manual of mental disorders. In: ( $5^{\text {th }}$ edn), pp. 338-345.

5. Chidiac CW (2019) An update on the medical consequences of anorexia nervosa. Curr Opin Pediatr 31(4): 448-453.

6. De Simone G, Scalfi L, Galderisi M, Celentano A, Di Biase G, et al (1994) Cardiac abnormalities in young women with anorexia nervosa. $\mathrm{Br}$ Heart J 71(3): 287-292.

7. Goldberg SJ, Comerci GD, Feldman L (1988) Cardiac output and regional myocardial contraction in anorexia nervosa. J Adolesc Health Care 9(1): 15-21.

8. Casiero D, Frishman WH (2006) Cardiovascular complications of eating disorders. Cardiol Rev 14(5):227-231.

9. Sachs KV, Harnke B, Mehler PS, Krantz MJ (2016) Cardiovascular complications of anorexia nervosa: a systematic review. Int J Eat Disord 49(3):238-248

10. Neumarker KJ (1997) Mortality and sudden death in anorexia nervosa. Int J Eat Disord 21(3): 205-212.

11. Isner JM, Roberts WC, Heymsfield SB, Yager J (1985) Anorexia nervosa and sudden death. Ann Intern Med 102(1): 49-52.

12. Levy PT, Machefsky A, Sanchez AA, Patel MD, Rogal S, et al. (2016) Reference ranges of left ventricular strain measures by two-dimensional speckle-tracking echocardiography in children: a systematic review and meta-analysis. J Am Soc Echocardiogr 29(3): 209-225.

13. Priori SG, Blomström-Lundqvist C, Mazzanti A, Blom N, Borggrefe $M$ et al. (2015) ESC Scientific Document Group. 2015 ESC Guidelines for the management of patients with ventricular arrhythmias and the prevention of sudden cardiac death: The Task Force for the Management of Patients with Ventricular Arrhythmias and the Prevention of Sudden Cardiac Death of the European Society of Cardiology (ESC). Eur Heart J 36(41): 2793-2867.

14. Devereux RB, Alonso DR, Lutas EM, Gottlieb GJ, Campo E, et al. (1986) Echocardiographic assessment of left ventricular hypertrophy: comparison to necropsy findings. Am J Cardiol 57(6): 450-458.

15. Lang RM, Badano LP, Mor-Avi V, Afilalo J, Armstrong A, et al. (2015) Recommendations for Cardiac Chamber Quantification by Echocardiography in Adults: An Update from the American Society of Echocardiography and the European Association of Cardiovascular Imaging. Eur Heart J Cardiovasc Imaging 16(3): 233-270.

16. Nagueh SF, Smiseth OA, Appleton CP, Byrd BF, Dokainish H, et al. (2016) Recommendations for the Evaluation of Left Ventricular Diastolic Function by Echocardiography: An Update from the American Society of Echocardiography and the European Association of Cardiovascular Imaging. Eur Heart J Cardiovasc Imaging. 17(12):1321-1360.

17. Hoek HW, Hoeken VD (2003) Review of the prevalence and incidence of eating disorders. Int J Eat Disord 34(4): 383-396.

18. Papadopoulos FC, Ekbom A, Brandt L, Ekselius L (2009) Excess mortality, causes of death and prognostic factors in anorexia nervosa. Br J Psychiatry 194(1): 10-17.

19. Regar P, Lachelin FM, Mimmik AM (2017) Initial treatment seeking from professional health providers for eating disorder: a review and synthesis of potential barriers and facilitators of first contact. Int J Eat Disord 50(3): 190-209.

20. Mehler PS, Blalock DV, Walden K, Simrat K, McBride J, et al. (2018) Medical findings in 1,026 consecutive adult inpatient-residential eating disordered patients. Int J Eat Disord 51(4): 305-313.

21. St John Sutton MG, Plappert T, Crosby L, Douglas P, Mullen J, et al. (1985) Effects of reduced left ventricular mass on chamber architecture, load, and function: a study of anorexia nervosa. Circulation 72(5): 991-1000.

22. Kastner S, Salbach-Andrae H, Renneberg B, Pfeiffer E, Lehmkuhl U, et al. (2012) Echocardiographic findings in adolescents with anorexia nervosa at beginning of treatment and after weight recovery. Eur Child Adolesc Psychiatry 21(1): 15-21.

23. Morris R, Prasad A, Asaro J, GuzmanM, Sanders L, et al. (2017) Markers of cardiovascular dysfunction in adolescents with anorexia nervosa. Glob Pediatr Health 4: 2333794X17727423.

24. Galetta F, Franzoni F, Cupisti A, Morelli E, Santoro G, et al. (2005) Early detection of cardiac dysfunction in patients with anorexia nervosa by tissue Doppler imaging. Int J Cardiol 101(1): 33-37.

25. Rossi MA, Zucoloto S (1982) Ultrastructural changes in nutritional cardiomyopathy of protein-calorie malnourished rats. Br J Exp Pathol 63(3): 242-253.

26. Abel RM, Grimes JB, Alonso D, Alonso M, Gay WA, et al. (1979) Adverse hemodynamic and ultrastructural change in dog hearts subjected to protein- calorie malnutrition. Am Heart J 97 (6): 733-744.

27. Inagaki T, Yamamoto M, Tsubouchi K, Miyaoka T, Uegaki J, et al. (2003) Echocardiographic investigation of pericardial effusion in a case of anorexia nervosa. Int J Eat Disord 33(3): 364-366.

28. Han HC, Ha FJ, Teh AW, Calafiore P, Jones EF, et al. (2018) Mitral Valve Prolapse and Sudden Cardiac Death: A Systematic Review. J Am Heart Assoc 7(23): e010584. 


\section{Your next submission with Juniper Publishers will reach you the below assets}

- Quality Editorial service

- Swift Peer Review

- Reprints availability

- E-prints Service

- Manuscript Podcast for convenient understanding

- Global attainment for your research

- Manuscript accessibility in different formats

( Pdf, E-pub, Full Tsext, Audio)

- Unceasing customer service

Track the below URL for one-step submission https://juniperpublishers.com/online-submission.php 